\title{
Philonsorbonne
}

14 | 2020

Année 2019-2020

\section{Délire, croyance et non-sens}

\section{Mathieu FRÈREJOUAN}

\section{(2) OpenEdition}

Journals

Édition électronique

URL : https://journals.openedition.org/philonsorbonne/1512

DOI : 10.4000/philonsorbonne.1512

ISSN : 2270-7336

\section{Éditeur}

Publications de la Sorbonne

\section{Édition imprimée}

Date de publication : 14 février 2020

Pagination : 179-202

ISSN : 1255-183X

\section{Référence électronique}

Mathieu FRĖREJOUAN, «Délire, croyance et non-sens », Philonsorbonne [En ligne], 14 | 2020, mis en ligne le 03 mars 2020, consulté le 09 juin 2021. URL : http://journals.openedition.org/philonsorbonne/ 1512 ; DOI : https://doi.org/10.4000/philonsorbonne.1512 


\title{
Délire, croyance et non-sens
}

\author{
Mathieu FRÈREJOUAN
}

\section{Introduction}

Il est devenu de plus en plus courant, en philosophie de l'esprit, de renouveler d'anciens débats portant sur la nature des états mentaux en se tournant vers leur contrepartie pathologique. C'est notamment le cas de la croyance que l'on interroge, depuis peu, à partir de la notion proprement psychopathologique de délire. Ce qui est alors en jeu pour le philosophe, comme l'observe à ce propos Lisa Bortolotti, ce n'est pas le délire en tant que tel mais bien plutôt le «débat concernant les critères d'attribution des croyances $\gg{ }^{1}$. À l'origine de ce débat se trouve la thèse de Donald Davidson suivant laquelle nous ne pouvons attribuer une croyance à un tiers qu'à condition que ce qu'il dit ou fait obéisse à certaines normes de rationalité $^{2}$. Or c'est précisément dans la mesure où le délire contredit, ou semble contredire, ces normes, que la question se pose de savoir s'il est possible de caractériser ce dernier comme une croyance. Ce faisant, à la racine de ce débat se trouve donc une certaine définition du délire comme ce qui contredit, ou semble contredire, nos normes de rationalité. Ce qui pose la question de savoir ce en quoi consistent ces normes. Selon Bortolotti, on peut distinguer la cohérence doxastique (la non-contradiction entre une croyance et les autres croyances du sujet), la sensibilité aux faits (la non-

1. Lisa Bortolotti, Delusions and Other Iirrational Beliefs, Oxford, New York, Oxford University Press, 2010, p. 1, notre traduction.

2. Voir Donald Davidson, Problems of Rationality, Oxford, New York, Oxford University Press, 2004, essays 11 et 12 . 
contradiction entre une croyance et les faits dont dispose le sujet) et la conséquence (la non-contradiction entre une croyance et le comportement qui lui est associé). De ces normes de rationalité, on peut alors déduire une définition négative du délire comme ce qui est incohérent, incorrigible et inconséquent.

Cependant, si le débat philosophique sur la nature «doxastique » du délire n'évoluait qu'au sein de ce cadre théorique, la question de savoir si des croyances délirantes sont possibles semblerait étrangement circulaire. En effet, si l'on admet la non-conformité du délire à nos normes de rationalité, on ne saurait parler de croyances délirantes et, inversement, si l'on admet sa conformité à ces mêmes normes, alors on ne saurait parler de croyances délirantes. Dès lors, la question de savoir si l'on peut attribuer une croyance à un sujet délirant a des allures de faux problème, qui se dissipe dès lors qu'on admet que, par définition, le délire n'est pas une croyance. Si la plupart des philosophes ne se satisfont pas d'une telle réponse c'est parce que, comme l'observe John Campbell, ce que l'on nomme généralement « délire » consiste dans " des assertions tout à fait sincères, faites par des personnes qui semblent comprendre ce qu'elles disent», qui ne sauraient donc être réduites à des «discours vides» $»^{3}$. Ce par quoi il faut comprendre que si l'on admet que le délire n'est pas une croyance, on se heurte alors à l'impossibilité de porter un tel jugement dans un contexte psychopathologique concret. Comme l'observe à ce propos Pascal Engel, si l'on refusait au délire le statut de croyance alors «les disciplines qui s'intéressent à la pathologie de la croyance [...] seraient impossibles » ${ }^{4}$, ce qui reviendrait à adopter l'attitude du philosophe «qui s'intéresse aux conditions de possibilité de la rationalité en général, et semble vouloir dicter au praticien ses propres critères $»^{5}$. Dès lors, un présupposé central $\mathrm{du}$ débat qui occupe la philosophie de l'esprit est que, d'un point de vue psychopathologique, l'attribution de délire implique l'attribution de croyance. Et c'est en effet une fois admis ce présupposé, et la nécessité pour le philosophe de prendre en compte les pratiques du psychologue et du psychiatre, que surgit le problème de savoir comment le délire peut être considéré, en même temps, comme contraire à nos normes de rationalité et comme une croyance.

Notre propos, dans cet article, sera de mettre en cause ce présupposé en montrant que l'attribution d'un délire n'implique pas nécessairement l'attribution d'une croyance. Et ce, non pas parce que le philosophe serait autorisé à «dicter au praticien ses propres critères »" mais parce que considérer ce que dit une personne comme « délirant » n'implique pas qu'il

3. John Campbell, «Rationality, Meaning, and the Analysis of Delusion », Philosophy, Psychiatry, and Psychology, 8-2-3, 2001, p. 91, notre traduction.

4. Pascal Engel, «Peut-on parler de croyances délirantes?», in Clinique de l'intentionnalité, 2001, p. 158.

5. Ibid., p. 158.

6. Ibid., p. 158 . 
s'agisse là d'une croyance mais seulement, comme nous le défendrons, d'un énoncé qui possède l'apparence d'une croyance. Pour ce faire, nous nous tournerons vers le dernier ouvrage de Wittgenstein, De la certitude, où ce dernier différencie ce que l'on nomme ordinairement "croyance » et " délire ». Comme l'observe en effet le philosophe, on semble devoir faire une « différence entre erreur (Irrtum) et délire (Geistesstörung)», différence qui pourrait se résumer au fait que « [1']erreur n'a pas seulement une cause, mais aussi un fondement $\gg^{7}$. Mais ce qui mérite alors d'être souligné, ce n'est pas tant la distinction faite par Wittgenstein, qui est somme toute banale, que la nature de cette distinction. Comme le précise par la suite le philosophe autrichien, la question de savoir quelle est la «différence entre erreur et délire » est avant tout celle de savoir «comment se présente cette différence quand je traite quelque chose comme erreur et quand je le traite comme dérangement mental $»^{8}$. Autrement dit, ce qui intéresse ici Wittgenstein, c'est ce qui distingue nos usages des termes de « délire » et de " croyance », c'est-à-dire la différence grammaticale qui les sépare. De ce point de vue, observer qu'on ne saurait confondre « croyance » et « délire » ce n'est pas mettre en lumière une différence entre des phénomènes, mais simplement rappeler que les usages que nous faisons de ces mots ne signifient pas (et ne peuvent signifier) la même chose. Comme l'observe à ce propos Vincent Descombes, le propre de la démarche grammaticale est d'arriver à « une réponse qui paraît triviale et qui, en effet, est triviale », de sorte que son objet n'est «pas vraiment cette réponse (nous la connaissons déjà), mais un moyen de l'accepter comme la bonne réponse ${ }^{9}$. C'est pourquoi notre tâche consistera, dans cet article, à lever ce qui peut faire obstacle au constat trivial suivant lequel le «délire» n'est pas, et ne peut être, une « croyance ».

Pour ce faire nous commencerons (1) par présenter les termes du débat tel qu'il est posé en philosophie de l'esprit, en introduisant son objet, le Syndrome de Capgras, et le modèle dominant, à savoir l'approche doxastique et empirique du délire. Nous interrogerons ensuite (2) ce que $D e$ la certitude peut apporter à ce débat, en soulignant que là où il est question de délire il ne saurait être question de croyance. En nous éloignant des écrits de Wittgenstein, nous tenterons enfin (3) de montrer, contre les critiques traditionnellement adressées au «non-doxasticisme», qu'un tel constat n'implique nullement de réduire le délire à un « acte de langage vide», dans la mesure où, si le sujet délirant ne saurait «croire ce qu'il dit», il peut néanmoins « croire qu'il le croit».

7. Ludwig Wittgenstein, De la certitude, Paris, Gallimard, 2002, § 73-74, p. 44.

8. Ibid., § 73, p. 44.

9. Vincent Descombes, Le Complément de sujet : enquête sur le fait d'agir de soi-même, 2018, p. 12. 


\section{Le Syndrome de Capgras : psychiatrie, neuropsychologie et philosophie}

\subsection{Le délire de Mme de Rio-Branco}

Comme nous l'avons vu, ce qui fait la singularité du débat sur la nature doxastique du délire est que, tout en s'enracinant dans le problème philosophique des relations entre croyance et rationalité, son objet est proprement empirique et psychopathologique. C'est pourquoi il est nécessaire, avant de nous pencher sur la conception doxastique du délire, de préciser de quoi, ou plutôt de qui, il est question lorsque nous parlons de "délire». De ce point vue, un mérite incontestable de la philosophie analytique est d'avoir délimité son objet en centrant le débat sur ce que l'on nomme le «Syndrome de Capgras ». À l'origine de cette appellation se trouve une présentation de malade, faite en 1923 par Joseph Capgras et Jean Reboul-Lachaux, devant la Société Clinique de Médecine Mentale. Ce qui fait la singularité de la patiente en question, « Mme de Rio-Branco », c'est la thématique qui domine ses idées délirantes, à savoir ce que les psychiatres nomment alors «l'illusion des "sosies" ». Comme l'observent ces derniers, Mme de Rio-Branco "métamorphose depuis une dizaine d'année chaque personne de son entourage et même ses plus proches, comme son mari et sa fille, en sosies différents, successifs et nombreux $»^{10}$. Et c'est de fait la plainte qui revient de manière récurrente dans la bouche de Mme de RioBranco elle-même : " au fur et à mesure qu'ils m'enlevaient une enfant, ils m'en donnaient une autre qui lui ressemblait... J'en ai eu plus de deux mille en cinq ans : ce sont des sosies $\gg{ }^{11}$.

Si un tel discours est assurément étrange, on notera que cela ne suffit pas à lui conférer le statut de "délire » au sens donné par les philosophes, c'est-à-dire comme contredisant nos normes de rationalité. Et de fait, aussi peu probable que puisse être une telle substitution, elle n'en semble pas moins possible. Ce qui fait alors l'étrangeté de "l'illusion des sosies » c'est, comme le soulignent Capgras et Reboul-Lachaux, qu'elle diffère de la «fausse reconnaissance ordinaire », puisque "c'est une seule et même personne qui se transforme successivement en premier sosie, en second, en troisième, etc., à quelques heures, quelques jours ou quelques semaines d'intervalle $»^{12}$. Ce que Mme de Rio-Branco semble croire, c'est donc qu'il s'agit de sosies tout en reconnaissant qu'il s'agit en apparence des mêmes personnes, et donc sans pouvoir justifier sa croyance. Le sosie est ainsi, comme le disent Capgras et Reboul-Lachaux citant Verlaine, une personne «qui n'est chaque fois ni tout à fait la même, ni tout à fait une autre »" C'est précisément cette contradiction entre la certitude que manifeste

10. Joseph Capgras et Jean Reboul-Lachaux, «L'illusion des "sosies" dans un délire systématisé chronique », Bulletin de la société clinique de médecine mentale, 11, 1923, p. 6.

11. Ibid., p. 9.

12. Ibid., p. 13, nous soulignons.

13. Ibid., p. 13. 
Mme de Rio Branco et l'absence totale de justification qui fait le caractère proprement délirant de son discours, ce dernier étant tout à la fois incohérent (en ce qu'il contredit le reste de ses croyances) et incorrigible (puisqu'il se trouve en-deçà de toute correction possible).

\subsection{Délire monothématique et expériences de pensées}

Une fois dit cela, il importe d'observer que, lorsque le Syndrome de Capgras est abordé en philosophie, ce n'est pas du cas de Mme de RioBranco qu'il est question. Et pour cause, le délire de cette dernière, loin de se limiter à « l'illusion des sosies », déborde « largement le milieu familial [...] pour s'étendre à sa maison, au monde entier $»^{14}$, et va jusqu'à inclure la patiente elle-même qui pense avoir été transformée puis remplacée («j'étais blonde, ils m'ont rendu châtain; j'avais les yeux trois fois comme je les ai $\gg^{15}$ ). Or lorsqu'il est question du Syndrome de Capgras en philosophie, l'objet de l'enquête se cantonne seulement à l'illusion des sosies considérée comme un délire «circonscrit» et donc "monothématique». En réalité, ce que les philosophes nomment le Syndrome de Capgras n'est pas tant le phénomène décrit par la sémiologie psychiatrique de la fin du $\mathrm{XIX}^{\mathrm{e}}$ siècle que celui étudié par la neuropsychologie cognitive contemporaine. Or celleci possède des enjeux épistémologiques qui lui sont propres, à savoir une approche des troubles mentaux centrées sur les "symptômes » plutôt que sur les «syndromes». Comme l'observe à ce propos Denis Forest, traditionnellement la frontière entre neurologie et psychiatrie, au moins depuis la fin du XIX ${ }^{\mathrm{e}}$ siècle, correspond à la différence que l'on peut faire entre les "pathologies $d u$ mental» (c'est-à-dire des troubles circonscrits dont on peut observer les corrélations avec des lésions cérébrales ellesmêmes déterminées) et les «pathologies mentales » (où c'est la subjectivité du patient, prise dans sa totalité, qui serait atteinte) ${ }^{16}$. C'est en suivant la même ligne de partage qu'on admet, parfois, l'existence d'une différence entre la neuropsychologie qui se préoccuperait seulement de symptômes, compris comme des troubles isolés, et la psychiatrie qui seule pourrait rendre compte des syndromes, compris comme l'organisation de différents symptômes entre eux. Et c'est précisément cette notion de syndrome, dans sa différence avec celle de symptôme, que la neuropsychologie cognitive entend mettre en cause.

En ce qui concerne le Syndrome de Capgras, cette rupture épistémologique s'observe avant tout dans les travaux d'Andrew Young qui constitue la principale référence (si ce n'est la seule) des philosophes de l'esprit. En effet, selon ce dernier une «découverte fondamentale de la neuropsychologie cognitive est que les déficits psychologiques acquis peuvent être hautement spécifiques » de sorte que l'on doit écarter

14. Ibid., p. 9 .

15. Ibid., p. 8.

16. Denis Forest, «La frontière entre psychiatrie et neurosciences », Les Cahiers du Centre Georges Canguilhem, n² 2-1, 2008, p. 147-173. 
«l'hypothèse, tenue pour acquise, de syndromes neuropsychologiques distincts $\gg{ }^{17}$. Pour qu'une telle assertion puisse s'appliquer aux troubles délirants, le neuropsychologue doit alors montrer l'indépendance du délire par rapport au reste des symptômes. C'est ce qui explique l'intérêt de Young pour le Syndrome de Capgras, dont la prévalence est pourtant particulièrement faible ${ }^{18}$, plutôt que pour des pathologies mentales plus répandues telles que la schizophrénie. Comme l'observe en effet ce dernier, si «les délires schizophréniques peuvent être florides et étendus, avec des patients qui semblent répondre par un nouveau délire à chaque question $\rangle^{19}$, le Syndrome de Capgras se présente au contraire comme « monothématique et souvent circonscrit; en dehors de la thématique du délire lui-même, les patients semblent tout à fait rationnels et parfaitement conscients de la réalité $»^{20}$. Autrement dit, avec le Syndrome de Capgras le syndrome est absorbé par le symptôme puisque «le syndrome supposé est largement défini par un seul symptôme (le délire lui-même) $»^{21}$.

Si ce bref détour par des questions qui concernent l'histoire et l'épistémologie de la psychopathologie nous semble nécessaire, c'est dans la mesure où le caractère «circonscrit» du Syndrome de Capgras n'est pas seulement déterminant pour la neuropsychologie mais aussi pour la philosophie de la psychopathologie. Et pour cause, si les philosophes souhaitent interroger la question de l'attribution des croyances à partir du délire, une condition sine qua non de ce questionnement est de pouvoir délimiter leur objet. De ce point de vue, le fait que «en dehors de la thématique du délire lui-même, les patients semblent tout à fait rationnels et parfaitement conscients de la réalité ${ }^{22}$ permet au philosophe de déterminer ce dont il parle. Mais de ce point de vue, on se gardera de croire qu'il appartient, par essence, au Syndrome de Capgras d'être « monothématique ». Comme nous l'avons vu, le cas princeps qu'est le délire persécutoire de Mme de Rio-Branco n'a rien de monothématique, ce qui pose la question de savoir quels délires nous devons retenir comme étant authentiquement des Délires de Capgras. En réalité, comme le reconnait Young lui-même,

17. Tony Stone et Andrew W. Young, « Delusions and Brain Injury: The Philosophy and Psychology of Belief », Mind \& Language, 12-3-4, 1997, p. 331, nous soulignons.

18. C'est ainsi, par exemple, que dans l'étude de Lut Tamam, Gonca Karatas, Tayfun Zeren et Nurgul Ozpoyraz («The prevalence of Capgras syndrome in a university hospital setting », Acta Neuropsychiatrica, 15-5, 2003, p. 290-295), sur une population clinique de 920 patients seul 1,3\% (12 patients) présentent les symptômes propres au « Syndrome de Capgras ». Qui plus est, parmi ces 12 patients les symptômes propres au Syndrome de Capgras accompagnent un diagnostic de « schizophrénie paranoïde », de « schizophrénie » (pour l'un d'entre eux), de «trouble bipolaire», de «trouble schizoaffectif» et «psychose organique ». En d'autres termes, on ne trouve aucun « délire monothématique » (à l'exception peut-être d'un des cas de «psychose organique ») qui est, nous le verrons, une idéalisation du Syndrome de Capgras plutôt qu'une réalité clinique.

19. Stone \& Young, op. cit., p. 329.

20. Ibid., p. 329.

21. Ibid., p. 331.

22. Ibid., p. 329. 
considérer le Syndrome de Capgras comme un délire monothématique n'est finalement rien d'autre qu'une «idéalisation, qui vise à éclairer des différences qui se répartissent probablement sur un continuum ${ }^{23}$. En d'autres termes, ce qui importe pour la neuropsychologie cognitive, c'est avant tout de montrer l'indépendance en droit du Syndrome de Capgras par rapport aux autres symptômes, en laissant de côté la question de savoir si un délire purement monothématique existe.

Ce point mérite d'être souligné car il détermine aussi la manière dont, implicitement ou explicitement, le problème de la nature doxastique du délire est posé en philosophie. Comme l'observait à cet égard Daniel Dennett, avant même de se tourner vers la psychopathologie, les philosophes avaient déjà "inventé des cas de fausses identités tirés par les cheveux pour illustrer leurs différentes théories philosophiques $»^{24}$. Cependant, ces expériences de pensées, aussi invraisemblables qu'elles puissent paraitre, trouvent un écho dans « le cas réel du Syndrome de Capgras qui a échappé jusque-là à l'attention des philosophes $\gg{ }^{25}$. Dès lors, tout se passe comme si les expériences de pensée, couramment invoquées en philosophie, s'étaient réalisées sous la forme de troubles psychopathologiques. Cependant, comme nous l'avons vu, une telle conclusion doit être nuancée dans la mesure où le "Syndrome de Capgras" doit lui-même se concevoir comme une « idéalisation » qui a ceci de commun avec les expériences de pensée qu'elle implique de mettre entre parenthèses certaines circonstances. Une telle observation ne vise nullement à discréditer ce qui a pu être dit, en neuropsychologie ou en philosophie, du Syndrome de Capgras. Ce que nous souhaitons souligner, et que l'on peut être enclin à oublier, c'est avant tout que le débat philosophique autour de la question de savoir si une délire est une croyance ne vise pas à déterminer si Mme de Rio-Branco (ou n'importe quel autre patient) croyait ce qu'elle disait. Ce qu'il s'agit de déterminer, c'est avant tout s'il est possible d'imaginer un contexte idéal où il y aurait un sens à croire que quelqu'un a été remplacé par un sosie, et ce en l'absence de toute justification rationnelle. C'est à cette question, et à cette question seulement, que nous tenterons de répondre.

\subsection{L'hypothèse de l'expérience anormale}

S'il existe différentes conceptions doxastiques du délire, c'est néanmoins le modèle dit «empiriste» qui domine actuellement la philosophie de l'esprit ${ }^{26}$. Bien que cette appellation ait été forgée par des

\section{Ibid., p. 329, nous soulignons.}

24. D. C. Dennett, Kinds of Minds: Toward an Understanding of Consciousness, New York, BasicBooks, 1996, p. 111, notre traduction.

25. Ibid., p. 112.

26. Un argument en faveur du doxastisme, qui n'est pas « empiriste», mais reste compatible avec une telle approche, consiste à mettre en évidence que nos propres croyances n'obéissent pas toujours aux critères de rationalité tels qu'ils sont définis par une certaine philosophie. Voir, à ce propos, Lisa Bortolotti, op. cit. 
philosophes, elle trouve encore son origine dans des modèles relevant de la psychopathologie cognitive. C'est ainsi que l'on doit au psychologue Brendam Maher d'avoir proposé l'hypothèse dite de «l'expérience anormale ». Contre l'hypothèse d'un déficit cognitif, Maher propose l'hypothèse suivant laquelle le délire ne serait pas un «phénomène primaire ", impliquant un échec du raisonnement, mais la "conséquence secondaire» d'une expérience anormale. Suivant une telle approche, «le contenu de la croyance délirante est déterminé directement par la nature et le contenu de l'hallucination $»^{27}$ (ou, nous y reviendrons, de n'importe quelle autre « expérience anormale »). On considère alors cette approche comme "empiriste" dans la mesure où la vérité de la croyance est évaluée relativement à l'expérience du sujet délirant, qui joue ainsi le rôle de norme. $\mathrm{Si}$, de ce point de vue, le délire est incorrigible, c'est dans la mesure où le témoignage d'autrui, qu'il vienne du médecin ou des proches, n'a pas d'autorité sur le témoignage des sens. C'est pourquoi, nous dit Maher, il est vain de dire au sujet délirant que sa croyance est fausse « car, dans la mesure où l'expérience sensorielle est primaire, elle ne peut être éliminée en lui opposant des faits contraires $»^{28}$. Et, de même, si le délire peut sembler contredire le reste des croyances du sujet, c'est simplement parce que le reste de ses croyances résulte d'un apprentissage passé qui est aussi secondaire par rapport à ce dont il fait l'expérience ici et maintenant.

Si Maher ne précise pas à quel type de délire s'applique l'hypothèse de «l'expérience anormale», celle-ci a été toutefois appliquée par Young au Syndrome de Capgras. Selon le neuropsychologue, on peut comprendre la croyance du sujet délirant suivant laquelle ses proches ont été remplacés par des sosies en faisant l'hypothèse d'une "prosopagnosie inversée ». Ce que l'on nomme " prosopagnosie » est un trouble neurologique où, à la suite d'une lésion cérébrale, le sujet devient incapable de reconnaitre visuellement les visages familiers. Cependant, précise Young, le prosopagnosique continue d'éprouver l'émotion associée à ces visages familiers, qui constitue ainsi une "réponse autonomique », indépendante de ce qu'il reconnaît consciemment $^{29}$. Ce que propose alors Young, c'est de comprendre Capgras comme «le reflet de la prosopagnosie $»^{30}$, où le sujet resterait capable de reconnaitre les traits du visage mais incapable d'éprouver l'émotion ordinairement associée à sa perception. En effet, «si quelqu'un perdait ces réponses autonomiques, on pourrait alors imaginer que les visages lui sembleraient bizarres, différents, pas comme il faut ${ }^{31}$. C'est cette

27. Brendan A. Maher, "The relationship between delusions and hallucinations ", Current Psychiatry Reports, 8-3, 2006, p. 182, notre traduction.

28. Ibid., p. 181.

29. Précisons que le prosopagnosique ne croit nullement, pour cette raison, que les personnes qu'ils voient ne sont pas ceux qu'ils disent être. Il s'agit là d'un trouble neurologique qui entraine certes une altération de la perception visuelle mais qui ne s'accompagne, habituellement, d'aucune forme de délire.

30. Stone \& Young, op. cit., p. 575.

31. Ibid., p. 575. 
perception anormale d'une personne comme n'étant « ni tout à fait la même, ni tout à fait une autre $»^{32}$ qui expliquerait alors la croyance délirante suivant laquelle nos proches ont été remplacés par des sosies.

Ce que l'on reproche traditionnellement à une telle hypothèse est de ne pouvoir combler l'écart qui sépare alors l'expérience anormale (le sentiment d'étrangeté éprouvé lors de la perception de visages familiers) et la croyance délirante (croire que ses proches ont été remplacés par des imposteurs). Et pour cause, en dehors de toute considération clinique, on peut observer que l'absence du « sentiment de familiarité » implique certes de voir les visages familiers comme nous étant étrangers, mais nullement de croire que nos proches sont des imposteurs. Or en l'absence d'une telle identité entre " voir » et « croire », l'hypothèse empiriste ne peut expliquer l'incorrigibilité et l'incohérence de ce dernier. On devrait, par exemple, pouvoir convaincre le sujet délirant du fait que, s'il perçoit le visage de sa fille comme lui étant étranger, cela ne signifie nullement qu'il s'agit d'un sosie. Ce qui revient à dire qu'on ne saurait considérer l'expérience anormale comme étant douée d'une normativité intrinsèque telle qu'on pourrait juger toute croyance comme devant en dériver. C'est sur ce point que les philosophes empiristes prennent le relais de la neuropsychologie, en tentant de combler cet écart entre "voir» et "croire ». On doit ainsi à Elisabeth Pacherie d'avoir proposé une distinction entre un empirisme de "l'explication» (explanationist) et un empirisme de "l'intégration » (endorsement), afin de répondre à ces objections. Ce que la philosophe présente comme un modèle " explicatif», c'est précisément l'hypothèse défendue par Maher suivant laquelle «le délire est adopté afin d'essayer d'expliquer une expérience anormale $»^{33}$. Comme nous l'avons vu, pour le psychologue l'expérience anormale (éprouver un sentiment d'étrangeté en percevant un visage familier) est un phénomène «primaire», tandis que la croyance délirante (croire qu'il s'agit d'un sosie) est un phénomène "secondaire» qui vient, après coup, expliquer la perception vécue. C'est ce fossé entre expérience primaire et croyance secondaire que propose alors de combler l'approche « intégrative » du délire :

Selon les modèles de l'intégration, l'expérience est composée du contenu même du délire, de sorte que le patient délirant croit simplement - c'est-à-dire, intègre doxastiquement - le contenu de son état expérientiel, ou du moins quelque chose qui est très proche de son état expérientiel ${ }^{34}$.

32. Reboul-Lachaux \& Capgras, op. cit., p. 13.

33. Elisabeth Pacherie, «Perception, emotions and delusions: Revisiting the Capgras Delusion », in Tim Bayne et Jordi Fernández (dir.), Delusion and Self-Deception : Affective and Motivational Influences on Belief Formation, Hoboken, Taylor and Francis, 2011, p. 109, notre traduction.

34. Ibid., p. 109. 
Dès lors, ce que l'on considère habituellement comme faisant seulement partie du contenu de la croyance (notamment le fait que nos proches soient des imposteurs), doit être considéré comme ayant son origine dans le contenu même de l'expérience. Comme l'observe Pacherie, dans une perspective intégrative, « le patient voit la femme qu'il regarde (qui est sa femme) comme étant un imposteur (c'est-à-dire quelqu'un qui ressemble seulement à sa femme) $\gg{ }^{35}$. Ce qui revient à dire que l'imposture n'est pas seulement un caractère de la croyance mais bien de l'état expérientiel lui-même. On peut dès lors écarter les objections soulevées plus tôt. On ne saurait introduire un quelconque écart entre le "sentiment d'étrangeté » propre à la perception des visages familiers, et la croyance en l'existence de "sosies ", puisque les proches sont vus comme des sosies. Et l'on ne saurait davantage reprocher à l'empiriste de ne pouvoir rendre compte de l'indifférence du délire aux faits, puisque l'on rend ainsi à l'expérience anormale sa normativité, son contenu étant, dans le délire, immédiatement intégré par la croyance. On semble parvenir, par ce biais, à rendre compte du Syndrome de Capgras comme étant une croyance certes anormale, en tant qu'elle est enracinée dans une expérience anormale, mais qui reste malgré tout une croyance.

\section{Le délire à la lumière de Wittgenstein}

\subsection{De la croyance à la certitude}

Une fois présenté le Syndrome de Capgras, ainsi que son explication par les modèles doxastiques et empiristes, nous pouvons revenir à notre question initiale : l'attribution du délire implique-t-elle nécessairement l'attribution d'une croyance? Comme nous l'avions annoncé, la réponse que nous proposons de donner à cette question s'inspirera des derniers écrits de Wittgenstein sur l'épistémologie et sera, en tant que telle, grammaticale. Il ne s'agira donc pas de déterminer si ce que nous dit tel ou tel patient, que l'on aurait diagnostiqué comme présentant un Délire de Cotard, doit être considéré comme une "croyance » ou un «délire». Ce qu'il s'agit pour nous de mettre en évidence est bien plutôt que tout énoncé qui est considéré comme «délirant», parce qu'il contredit nos normes de rationalité, ne saurait être considéré comme une "croyance». Bien sûr, il se peut tout à fait que ce que dit un patient nous semble être, à première vue, « délirant » et se révèle être, après examen, une " croyance " seulement erronée. Comme l'observait à cet égard Eugen Bleuler, « souvent, des troubles sont simulés par le fait que l'examinateur et le patient ne parlent pas le même langage », lorsque « le patient prend dans un sens symbolique ce que le médecin entend au sens propre » ou, inversement, lorsqu'une «tournure figurée quelconque 
est prise au pied de la lettre par le patient ${ }^{36}$. Cependant, la question de savoir si ce que nous dit tel ou tel patient est une croyance que nous prenons pour un délire, ou un délire que nous prenons pour une croyance, est une question proprement empirique qui, en tant que telle, concerne le clinicien plutôt que le philosophe. Ce que nous souhaitons mettre en évidence, plus modestement, c'est que là où nous avons des raisons de considérer un énoncé comme délirant nous ne pouvons, dans ces mêmes circonstances, le considérer comme étant en même temps une croyance. En d'autres termes, l'attribution d'un «délire» ne peut impliquer l'attribution d'une « croyance ».

Si une telle impossibilité échappe parfois aux philosophes c'est, pour commencer, dans la mesure où nous sommes enclins à exagérer l'extension de la catégorie de croyance. On admet ainsi qu'un énoncé assertif tel que «cet enfant est ma fille» pourrait être paraphrasé sous la forme «je crois que cet enfant est ma fille». C'est précisément cette généralisation abusive de la notion de "croyance » que Wittgenstein s'efforce de dénoncer dans De la certitude. Le point de départ de sa réflexion est une série d'articles de G. E. Moore ${ }^{37}$ mettant en évidence certaines propositions dites du « sens commun », comme relevant du "savoir » et non, comme le suggère la philosophie traditionnelle, de la «croyance». C'est ainsi qu'au début de A Defense of Common Sense, Moore propose « une longue liste de propositions qui semblent être, à première vue, des truismes trop évidents pour être même énoncés ", propositions «dont je peux dire de chacune d'entre elle (selon nous) que je sais qu'elle est, de manière certaine, vraie $»^{38}$. Or si cette liste contient des propositions que les philosophes considèrent parfois comme logiquement nécessaires («il existe en ce moment un corps humain vivant, qui est mon corps »), on y trouve aussi des propositions que l'on pourrait être tenté de considérer comme empiriquement contingentes ( « [ce corps] depuis qu'il est né, a été en contact ou à proximité de la surface de la terre »). Et c'est précisément dans la mesure où l'on considère ces propositions comme susceptibles de vérité ou de fausseté que l'on peut croire être en droit de dire non seulement « je suis né sur la surface de la terre » mais « je crois être né à la surface de la terre ». On ajoutera que c'est bien dans cette catégorie que les philosophes sont enclins à ranger le Délire de Cotard, dans la mesure où la proposition «mes proches ont été remplacés par des sosies ", ne semble impliquer aucune impossibilité logique.

Ce que retiendra Wittgenstein de l'analyse de Moore, c'est avant tout que ces propositions ne sauraient être considérées comme de simples « croyances", dans la mesure où le fait d'en douter semble, dans des

36. Eugen Bleuler, Dementia praecox ou Groupe des schizophrénies, trad. fr. Alain Viallard, Paris ; Clichy, France, E.P.E.L. ; G.R.E.C., 1993, p. 103.

37. Il s'agit, plus précisément de «Proof of an external world», in Thomas Baldwin (dir.), Selected writings, London; New York, Routledge, 1993, p. 147-170 et «A defense of common sense », in Philosophical papers, Reprinted., London, Routledge, 2002, p. 32-60.

38. Moore, «A defense of common sense », op. cit., p. 32, notre traduction. 
circonstances ordinaires, dépourvu de sens. Cependant, à la différence $\mathrm{du}$ philosophe britannique, il ne considère pas pour autant qu'elles feraient l'objet d'une forme particulière de "savoir ». Comme l'observe Wittgenstein, "“Je le sais" veut souvent dire : J'ai pour mon énoncé des raisons qui sont justes» ce qui suppose que l'on "puisse se représenter comment on peut savoir quelque chose de cet ordre $»$. Dès lors, lorsque je dis «savoir» quelque chose, mon énoncé reste ouvert à la vérification, comme pour toute proposition susceptible de vérité ou de fausseté. Or ce qui fait la singularité des propositions dont Moore fait la liste est que, tout en semblant être susceptibles de vérité et de fausseté, elles n'en sont pas moins soustraites à toute forme de vérification. C'est ainsi, nous dit Wittgenstein, que jamais «dans la vie, je [ne] teste la vérité de la proposition qui dit que je sais qu'il y a là une main (plus précisément ma main) » ${ }^{40}$. Une telle proposition peut être vraie ou fausse (il peut s'agir d'une hallucination ${ }^{41}$ ) et pourtant il n'y aurait pas de sens, dans des circonstances normales, à la vérifier.

Certes, comme nous l'avons vu, on peut être tenté de soutenir que si aucune vérification n'est nécessaire ici, c'est dans la mesure où la proposition «il y a là une main» (ou autre) est la description d'une expérience immédiate. Autrement dit, si «dans la vie» nous ne vérifions jamais «qu'il y a là une main » c'est parce que la présence de ma main m'est donnée, immédiatement, par le toucher et par la vue. Cependant, comme l'observe Wittgenstein, on se heurte au fait que de nombreuses propositions sont soustraites à toute forme de vérification, sans impliquer pour autant une quelconque expérience. Il serait tout aussi absurde de vérifier, par exemple, «que j'ai eu des aïeux, que les gens qui se donnaient pour mes parents étaient réellement mes parents, etc. $»^{42}$. Ce que l'approche empiriste est ainsi parfois tentée d'oublier est que la proposition «les gens qui se donnaient pour mes parents étaient réellement mes parents » n'est pas, dans des circonstances normales, ouverte à la vérification. Mais, précisonsle, cela est aussi vrai des propositions qui semblent impliquer la description d'une expérience perceptuelle, comme lorsque nous disons « qu'il y a là une main ». Comme l'observe Wittgenstein, si « un aveugle me demandait : "Astu deux mains ?", ce n'est pas en regardant que je m'en assurerais ${ }^{43}$. Et

39. Wittgenstein, op. cit., § 18, p. 34.

40. Ibid., § 6, p. 32.

41. C'est l'argument classique du «membre fantôme » que l'on trouve déjà chez Descartes : «j'ai autrefois appris de quelques personnes qui avaient les bras et les jambes coupés, qu'il leur semblait encore quelquefois sentir de la douleur dans une partie qui leur avait été coupée ; ce qui me donnait sujet de penser, que je ne pouvais aussi être assuré d'avoir mal à quelqu'un de mes membres, quoique je sentisse en lui de la douleur » (René Descartes, Méditations métaphysiques: objections et réponses, suivies de quatre lettres, Paris, GFFlammarion, 1992, p. 183). Comme nous le verrons le fait qu'il s'agisse d'une expérience pathologique, se produisant dans des circonstances exceptionnelles, est bien sûr significatif.

42. Wittgenstein, op. cit., § 158, p. 61.

43. Ibid., § 125, p. 54. 
pour cause, « je ne sais pas pourquoi j'irais faire confiance à mes yeux si j'en étais à en douter ${ }^{44}$. Autrement dit, se référer à l'expérience c'est déjà entrer dans le jeu de la vérification. Ce faisant, la certitude que nous « avons deux mains » est antérieure à toute perception, de sorte qu'on ne saurait faire de l'expérience le fondement des propositions du sens commun.

\subsection{Certitude et logique}

Ce qui fait la normativité, dans certaines circonstances, d'une proposition telle que «les gens qui se donnaient pour mes parents sont réellement mes parents " doit donc être cherché ailleurs que dans l'expérience. Selon Wittgenstein, si certaines propositions sont soustraites à toute forme de vérification, c'est précisément parce qu'on ne saurait douter d'une proposition sans admettre, par là même, la certitude d'une autre. Comme le dit le philosophe autrichien, «les questions que nous posons et nos doutes reposent sur ceci : certaines propositions sont soustraites au doute, comme des gonds sur lesquels tournent ces questions et doutes $\gg{ }^{45}$. Je ne peux douter, par exemple, du fait que celui qui dit être mon père est bien mon père biologique, qu'à condition de tenir pour certain que $\mathrm{j}$ 'ai un « père " et que les hommes sont, en général, ce qu'ils disent être. Et c'est justement parce que la généralisation du doute au-delà de ces limites implique l'abolition de la possibilité même du doute qu'il est nécessaire que " certaines propositions [soient] soustraites au doute, comme des gonds sur lesquels tournent ces questions et doutes». C'est pourquoi de telles propositions ne sauraient relever de la « croyance », ou même du « savoir », mais doivent être considérées comme des «propositions qui jouent un rôle logique particulier dans le système de nos propositions empiriques $\gg{ }^{46}$. Dès lors, que «les gens qui se donnaient pour mes parents étaient mes parents » n'est pas quelque chose que « je crois », ou même que « je sais », mais une certitude dont la fonction est proprement logique ${ }^{47}$.

Soutenir ainsi que les propositions du sens commun «jouent un rôle logique " peut toutefois prêter à confusion, dans la mesure où l'on pourrait être tenté de rétorquer qu'il ne s'agit pas tant de règles logiques que de propositions empiriques qui, dans certaines circonstances, ont une fonction logique. Si je suis contraint de présupposer, dans des circonstances déterminées, que «les gens qui se donnaient pour mes parents sont mes parents », une telle proposition n'en reste pas moins, dans d'autres circonstances (celles, par exemple, d'une adoption), susceptible de vérité

44. Ibid., § 125, p. 54.

45. Ibid., § 340-341, p. 89.

46. Ibid., § 136, p. 56.

47. Danièle Moyal-Sharrock, dans Understanding Wittgenstein's On certainty (Basingstoke, Palgrave Macmillan, 2007), propose le concept de «certitude-gond (hinge-certainty) pour souligner le caractère proprement logique que possède ici le terme de «certitude ». Nous continuerons toutefois à employer simplement le terme de «certitude » (et non de « certitudegond »). 
et de fausseté. De ce point de vue, on semble devoir dire que «je crois que les gens qui se donnaient pour mes parents sont mes parents », puisqu'une telle proposition est susceptible de vérité ou de fausseté, mais qu'il s'agit toutefois d'une croyance qui, en tant qu'elle est simplement présupposée, échappe de fait à toute forme de vérification. Cependant, ce que Wittgenstein vise à montrer, dans De la certitude, c'est précisément que la fonction de ce type de propositions ne peut être comprise si on la réduit à de simples « présuppositions ».

Comme l'observe à ce propos Danièle Moyal-Sharrock, dans son commentaire de De la certitude, si l'on est enclin à penser que ces propositions restent des propositions empiriques, c'est dans la mesure où nous échouons à les distinguer de ce que la philosophe nomme leurs Doppelgänger ( « sosies »). Pour comprendre ce que signifie cette notion de Doppelgänger, qui sera déterminante lorsque nous reviendrons à la question du délire, il est nécessaire de noter, à l'instar de Moyal-Sharrock, que la « phrase » et la « proposition » n'ont pas, pour Wittgenstein, le même statut. Cette différence peut se résumer au fait qu'à une même phrase, comprise ici comme une simple suite de mots, peut correspondre différentes propositions selon sa signification, c'est-à-dire selon les circonstances dans lesquelles nous l'employons. On parlera alors de Doppelgänger lorsque nous avons des "phrases identiques mais possédant différents usages, et donc différents statuts " ${ }^{48}$. C'est ainsi qu'une même phrase, telle que «les gens qui se donnaient pour mes parents étaient réellement mes parents» peut être « employée de manière à avoir la fonction d'une règle de grammaire, d'une proposition empirique ou d'une expression spontanée $» 49$. On notera, à ce propos, que la notion de Doppelgänger ne décrit pas tant une catégorie à laquelle appartiendraient certaines propositions, qu'une relation entre certaines propositions, à savoir le fait d'être en apparence identiques tout ayant des significations différentes. Pour reprendre une expression de Wittgenstein, ce que les Doppelgänger ont en commun, c'est une " grammaire de surface » à savoir ce que, dans l'usage d'une phrase, " on peut saisir par l'oreille $\aleph^{50}$. De ce point de vue, une phrase est le Doppelgänger d'une autre si elle partage la même " grammaire de surface », ce qui n'implique nullement que ces phrases possèdent la même " grammaire profonde ».

Ce qu'il s'agit ainsi de mettre en évidence, c'est avant tout que s'il est possible de douter, dans certaines circonstances exceptionnelles, du fait que « nos parents sont parents », cela n'implique nullement que nous puissions étendre ce doute à des circonstances ordinaires. Car ce dont nous doutons, ce n'est nullement de notre certitude en tant que certitude, mais seulement d'une proposition empirique qui, en tant qu'elle est exprimée par les mêmes mots, est son Doppelgänger. Que l'on puisse douter du fait que nos parents

48. Ibid., p. 140-141, notre traduction.

49. Ibid., p. 140-141.

50. Wittgenstein, Recherches philosophiques, Paris, Gallimard, § 664, p. 237. 
sont ce qu'ils disent être dans les circonstances d'une adoption cachée (ou dans des circonstances encore plus exceptionnelles) ne saurait donc justifier, de quelque manière que ce soit, que nous puissions douter dans des circonstances ordinaires que nos parents sont ce qu'ils disent être. Cela nous permet tout au plus, nous y reviendrons, non pas de douter de ce que nous tenons pour certain, mais de croire que nous en doutons.

\subsection{De la certitude au délire}

Une fois mis en évidence l'écart qui sépare la «croyance» de la " certitude », la question se pose alors de savoir ce que nous attribuons à quelqu'un lorsque nous jugeons qu'il « délire ». S'agit-il d'une croyance, d'une certitude, ou encore d'autre chose ? On commencera par noter que lorsque De la certitude est invoquée en philosophie de la psychopathologie, c'est presque toujours pour reconnaitre au délire un statut symétriquement identique à celui de nos propres certitudes ${ }^{51}$. C'est le cas notamment du philosophe John Campbell qui s'appuie sur les derniers écrits de Wittgenstein pour opposer aux approches «empiristes» du délire une approche qu'il présente comme «rationaliste». Selon le philosophe américain, on pourrait en effet comprendre les délires comme des « certitudes » (ou, dans sa propre terminologie, des «propositions-cadres » (framework-propositions)), qui formeraient ainsi, chez le sujet délirant, les "présupposés d'arrière-fond qui sont nécessaires pour toute vérification de la justesse des propositions $»^{52}$. Ce n'est donc plus une «expérience anormale » qui se trouverait à l'origine des croyances du sujet délirant, comme le suggérait l'empiriste, mais une «certitude anormale ». Et de fait, si l'on suppose qu'une « certitude» du sujet délirant n'est pas, par exemple, «que les gens qui se donnaient pour mes parents étaient réellement mes parents» mais, au contraire, "que les gens qui se donnaient pour mes parents n'étaient pas réellement mes parents », alors ce que nous dit le sujet souffrant du Syndrome de Capgras semble prendre sens. Si ce dernier rejette toutes les preuves que lui apporte son entourage, voire même ce qu'il peut observer par lui-même, c'est parce que toutes ses croyances sont évaluées à l'aune de ses propres "propositions-cadres", qui endossent ici le rôle de norme. Ce faisant, selon l'approche rationaliste, l'attribution d'un délire n'implique pas tant l'attribution d'une «croyance » que d'une «certitude», dont l'incorrigibilité ne fait que refléter le statut logique de nos propres certitudes.

51. C'est le cas, nous le verrons, de John Campbell (op. cit.) mais aussi de John Rhodes et Richard G. T. Gipps, («Delusions, Certainty, and the Background », Philosophy, Psychiatry, \& Psychology, 15-4, 2009, p. 295-310). Pour une critique de cet usage de De la certitude, voir notamment Tim Thornton, "Why the idea of framework propositions cannot contribute to an understanding of delusions ", Phenomenology and the Cognitive Sciences, 7-2, 2008, p. $159-175$.

52. Campbell, op. cit., p. 96. 
Si une approche rationaliste du délire est assurément séduisante, en ce qu'elle semble mieux rendre compte de l'incorrigibilité du délire que ne le fait l'approche empiriste, la lecture de Wittgenstein sur laquelle elle s'appuie pose néanmoins problème. Et pour cause, en suggérant qu'attribuer un délire à quelqu'un, c'est lui attribuer d'autres certitudes, nous comprenons ces dernières, au moins implicitement, comme des états psychologiques. Ce point apparaît, sans ambiguïté, chez Campbell lui-même, qui présente le délire comme «une perturbation top-down des croyances fondamentales du sujet $»^{53}$. Mais c'est se méprendre sur le propos de Wittgenstein qui est, comme nous l'avons déjà souligné, d'ordre grammatical et donc logique. Comme le précise le philosophe autrichien, lorsqu'il dit «[qu']il y a certaines circonstances où l'homme ne peut pas se tromper ", "peut» est « employé logiquement, la proposition ne dit pas que dans ces circonstances 1 'homme ne peut rien dire de faux $»^{54}$. Or si d'un point de vue psychologique on peut être tenté d'attribuer des « certitudes » à un autre que soi, d'un point de vue logique une telle attribution est radicalement contradictoire ${ }^{55}$. Ce qui nous égare, ici encore, est que lorsque nous disons «cet homme est mon père », il est bien sûr possible d'imaginer des circonstances où le contraire pourrait être dit. Cependant, ce qui est alors contredit n'est pas la proposition que nous tenons pour certaine, mais une proposition empirique exprimée par la même phrase, son Doppelgänger. En revanche, que quelqu'un puisse croire le contraire de ce que nous tenons pour certain n'est pas seulement étrange, mais a priori exclu par la fonction logique qu'endossent nos certitudes.

Cet écart qui sépare une lecture psychologique d'une lecture grammaticale de De la certitude, nous le retrouvons aussi dans la manière dont nous devons comprendre le délire. Comme nous l'avons vu, dès lors qu'il est admis que les propositions du sens commun jouent un rôle logique, alors toute proposition qui les contredit devra être considérée comme un non-sens. Ce qui signifie, nous y reviendrons, qu'aucune compréhension $\mathrm{du}$ délire, en tant que non-sens, n'est possible. Au contraire, si l'on suit une approche psychologique du délire, telle qu'est du moins défendue par Campbell, on arrive à une toute autre conclusion. Certes, le philosophe reconnait sans peine "qu'une altération des principes-cadres (framework principles) entraîne de manière directe une altération de la signification des mots employés $\gg{ }^{56}$. Mais cela n'implique nullement, pour le philosophe américain, que les mots employés par le sujet délirant soient dépourvus de toute signification, seulement qu'ils possèdent une autre signification. On

\section{Ibid., p. 89.}

54. Wittgenstein, op. cit., § 155, p. 60.

55. Cette question touche de près à celle du «principe de charité » chez Quine qui est aussi débattu, en philosophie de la psychopathologie. Sur cette question voir notamment Manuel Rebuschi, Maxime Amblard et Michel Musiol, «Schizophrénie, logicité et perspective en première personne », L'Évolution Psychiatrique, 78-1, 2013, p. 127-141.

56. Campbell, op. cit., p. 98. 
observerait, de ce fait, un écart incommensurable entre notre langage et celui du sujet délirant, entrainant ainsi une situation d'incommunicabilité : «les nouvelles significations ne seraient pas même commensurables avec les anciennes significations; aucune traduction n'est possible entre les cadres (frameworks) ${ }^{57}$. Ce que l'on retrouve ainsi, sous l'approche rationaliste du délire, c'est alors ce que la psychopathologie phénoménologique a parfois présenté comme l'incompréhensibilité du délire. C'est, généralement, au psychiatre et philosophe Karl Jaspers qu'est attribuée cette notion, lequel observe en effet, dans sa Psychopathologie générale, que si «le système délirant (...) nous est tout à fait compréhensible dans tout son ensemble» il « devient incompréhensible pour nous [...] lorsqu'on atteint les sources mêmes des expériences primaires ${ }^{58}$. Et c'est bien dans cet esprit que s'inscrit Campbell, qui cite à ce propos (de manière assez vague) « un schizophrène » qui aurait dit que, lorsqu'il parle, « les mots possèdent deux significations : les significations qu'ils ont ordinairement et les significations qu'il tente d'exprimer lorsqu'il les emploie ${ }^{59}$. S'il est possible de comprendre les croyances du sujet délirant en elles-mêmes, le fait qu'elles s'ancrent dans des certitudes qui nous sont absolument étrangères exclut que nous puissions en comprendre la signification profonde.

Cependant si l'on admet, avec Wittgenstein, que nos certitudes ont un statut plutôt logique que psychologique, alors il ne faut plus dire de l'énoncé délirant les contredisant que l'on ne peut comprendre sa signification mais, au contraire, qu'il n'a pas de signification. Ce point apparaît clairement dans De la certitude, lorsque survient la question de savoir s'il est possible de douter de tout (question qui n'est pas étrangère à celle du Syndrome de Capgras, tel qu'il est pensé en philosophie de l'esprit) :

Il $\mathrm{y}$ a des cas où un homme donne des signes de doute (alors que nousmêmes ne doutons pas), des cas d'une nature telle que nous ne pouvons pas comprendre en toute assurance comme signes de doute les signes qu'il émet. C'est-à-dire : pour que nous comprenions comme tels ses signes de doute, il doit ne les donner que dans des cas déterminés et non dans d'autres ${ }^{60}$.

Ce que Wittgenstein désigne ici par «signe», ce sont avant tout des « signes écrits ou vocaux » ${ }^{61}$ qui composent les phrases sans en déterminer, par eux-mêmes, la signification. De ce point de vue, donner « des signes de doute (alors que nous-mêmes ne doutons pas)» revient simplement à prononcer une phrase telle que «je ne suis pas sûr que mes parents soient

57. Ibid., p. 98.

58. Karl Jaspers, Psychopathologie générale, trad. fr. Jacques Sédat, Paris, Bibliothèque des introuvables, 2000, p. 96.

59. Ibid., p. 98.

60. Wittgenstein, op. cit., $§ 154$, p. 60.

61. C'est ainsi que Wittgenstein observe par ailleurs, sans équivoque, que «en chinois, les signes vocaux ou écrits « $2 \times 2=4$ » pourraient avoir une autre signification ou être un nonsens patent » (Ibid., § 10, p. 33). 
mes parents ", dans des circonstances où nous n'avons aucune raison d'en douter. Or dans ces circonstances, et c'est là le point essentiel, il est clair pour Wittgenstein que « nous ne pouvons pas comprendre en toute assurance comme signes de doute les signes qu'il émet». Ce par quoi il faut comprendre que nous reconnaissons certes les mots prononcés comme devant exprimer le doute, mais aussi comme échouant à le signifier dans ces circonstances. Par conséquent, si la phrase prononcée contredit ce que nous tenons pour certain, alors elle n'est pas l'expression d'un sens qui se trouverait hors de notre portée, elle est une suite de signes qui tourne à vide. C'est pourquoi, à la différence des approches psychologiques ou phénoménologiques du délire, on ne dira pas de ces énoncés qu'ils sont " incompréhensibles », au sens où leur signification nous échapperait, mais plutôt qu'il n'y a rien à comprendre, dans la mesure où ils n'ont pas de signification. De ce point de vue, reconnaitre un énoncé comme " délirant» ce n'est pas lui reconnaitre le statut de «croyance» ou, encore moins, de « certitude ». C'est reconnaitre, simplement, qu'il n'a pas de sens.

\section{Non-sens et acte de langage vide}

\subsection{Le délire et ses Doppelgänger}

Comme nous venons de le voir, la différence grammaticale que met en évidence Wittgenstein entre «croyance» et «délire» est radicale : si l'on considère qu'il y a «délire » lorsqu'un énoncé contredit ce que nous tenons pour certain, alors ce dernier est simplement un «non-sens ». Cependant, on ne saurait se contenter d'une telle conclusion dans la mesure où elle soulève davantage de questions qu'elle n'apporte de réponses. Et pour cause, comme nous l'avons vu en introduction, ce qui est à l'origine du débat sur la nature doxastique du délire c'est la conviction, en philosophie de l'esprit, qu'on ne peut réduire le délire à un « acte de langage vide». C'est ainsi que, selon Engel, la thèse suivant laquelle le délire ne serait pas une croyance " est trop forte, ou erronée » car "dans notre pratique usuelle d'attribution de croyances, nous n'hésitons pas à attribuer aux gens des états contradictoires, irrationnels, ou même simplement bizarres ${ }^{62}$. Par suite, il semble «plus raisonnable de dire, au sujet de croyances du type ci-dessus, que ce sont des croyances, qui semblent avoir toutes les caractéristiques usuelles de ce type d'état, mais qui sont, en quelque façon, déviantes, anormales, ou pathologiques $\gg{ }^{63}$. Et il n'en va pas différemment chez Campbell qui, malgré sa critique du doxastisme, refuse néanmoins de voir le délire comme un « discours vide» (empty speech) car il s'agit après tout « d'assertions tout à fait sincères, faites par des personnes qui semblent comprendre ce qu'elles disent ${ }^{64}$. Qui plus est, les rares philosophes à avoir rappelé la portée

62. Engel, op. cit., p. 158.

63. Ibid., p. 158.

64. Campbell, op. cit., p. 91. 
logique des propos de Wittgenstein dans De la certitude, se contentent de dénoncer les usages plus ou moins sauvages qui en sont fait en philosophie de la psychopathologie, tout en refusant de se prononcer sur la question de savoir si le délire peut être considéré ou non comme une croyance ${ }^{65}$. Notre propos, dans cette dernière section, sera de montrer que ces appréhensions à l'égard de toute distinction radicale entre délire et croyance ne sont pas fondées (et elles sont, de fait, rarement justifiées). En réalité, dire que le délire est un "non-sens» ne contredit pas «notre pratique usuelle d'attribution de croyances », pas plus que cela n'implique de mettre en cause la « sincérité » du sujet délirant ou le fait qu'il « semble comprendre ce qu'il dit ». Cependant, force est de constater que les quelques remarques faites par Wittgenstein sur le délire dans De la certitude ne permettent pas de mettre cela en évidence ${ }^{66}$. Et ce qui fait défaut à l'analyse proposée par le philosophe autrichien c'est, il nous semble, de montrer que si l'attribution du délire ne saurait impliquer l'attribution d'une croyance, celles-ci n'en sont pas moins grammaticalement liées.

Pour mettre ce point en évidence il importe, avant tout, de distinguer le délire comme "non-sens » du délire compris comme " acte de langage vide ». Cette conception du délire a été défendue par German Berrios ${ }^{67}$ dont le propos parfois excessif sert souvent de faire-valoir aux tenants du doxastisme. En quelques mots, ce à quoi s'oppose avant tout Berrios c'est à l'opinion dominante suivant laquelle les délires seraient «des actes de langage sémantiquement chargés, et pouvant donc être classés comme des croyances "fausses" ou "pathologiques" $\gg{ }^{68}$. C'est oublier, nous dit ce dernier, que si le contenu du délire peut rappeler celui d'une croyance, sa forme exclut qu'on puisse établir un tel rapprochement. C'est pourquoi on doit concevoir les discours délirants comme «des actes de langage vides, dont le contenu informationnel n'est ni le monde, ni le soi. Ils ne sont l'expression symbolique de rien ${ }^{69}$. Si l'on admet volontiers que la conclusion à laquelle arrive Berrios pose problème, c'est avant tout l'opposition simpliste entre " actes de langage sémantiquement chargés » et «actes de langage vides» qui doit être remise en cause. Car, comme nous allons tenter de le montrer, soutenir que le «délire» n'est pas une « croyance» n'implique pas qu'il soit, pour cette raison, un «acte de langage vide $»$.

65. C'est le cas notamment de Tim Thornton (op. cit.) et de Rupert Read (« On approaching schizophrenia through Wittgenstein », Philosophical Psychology, 14-4, 2001, p. 449-475.).

66. Précisons que cette insuffisance vient seulement du fait que le «délire», dans De la certitude, a pour seule fonction de dénoncer la généralisation abusive que peuvent faire les philosophes de la notion de «croyance erronée ». Il serait absurde, de ce point de vue, d'exiger de Wittgenstein une analyse approfondie de ce qui n'est pas son objet.

67. G. E. Berrios, « Delusions as "wrong beliefs" : a conceptual history », The British Journal of Psychiatry. Supplement, 14, 1991, p. 6-13.

68. Ibid., p. 6, notre traduction.

69. Ibid., p. 12, nous soulignons. 
On peut, pour commencer, distinguer les énoncés délirants de ce que l'on nomme parfois la «jargonophasie » ou, de manière plus expressive, la « salade de mots » ${ }^{70}$, que l'on peut considérer à proprement parler comme des «actes de langage vides». On en trouve un exemple frappant dans une présentation de malade faite par Michel Cénac en 1923, où ce dernier se penche sur le cas de "Erneste C. », une patiente de 48 ans qui " converse à peu près normalement quand on parvient à fixer son attention » mais qui, livrée à elle-même, « emploie un langage totalement incohérent, entièrement néologique $»^{71}$. Cénac nous cite, dans la suite de sa présentation, un extrait des propos de «Erneste C.», qu'il présente comme une «logorrhée néologique » :

M. Stroben a une congestion interne, congestion étranglée, congestion jaune et toi tu les as en jaune - et gim. Et tous en jaune et en terne. De la terne de la tiendam - ça fait 24 ans que je le connais en terme - le gim de l'air de la dantam, le cim de l'air de grand terme en terme de la citadelle en citadelle - en strum de l'ombre de mon corps ${ }^{72}$.

Si nous pouvons parler ici «d'actes de langage vides » c'est d'abord dans la mesure où les sons prononcés par la patiente (" gim », «tiendam», « dantam », « cim», «strum», etc.) ne rappellent que de loin ce que l'on pourrait considérer comme des « mots ». Il s'agirait plutôt, comme l'observe Cénac, de ce que « des linguistes n'hésiteraient pas à qualifier de phonèmes, c'est-à-dire de mots n'ayant pas de sens et réduits à leur simple valeur phonétique $\gg{ }^{73}$. De ce point de vue, ce qui manque au langage d'Erneste pour faire sens, c'est le fait d'obéir aux règles morphologiques de la langue française. On notera toutefois que, même là où les mots employés ne sont pas de simples phonèmes, et où des débuts de phrases s'esquissent, le discours d'Erneste reste dépourvu de sens. C'est ainsi que des phrases telles que « de l'air de grand terme en terme de la citadelle en citadelle » obéissent certes aux règles de la morphologie, mais contreviennent cette fois aux règles de la syntaxe. Ces phrases semblent en effet obéir, comme l'observe Cénac, à «des lois d'associations inférieures, telles que l'assonance $»^{74}$, qui ne suffisent pas à faire émerger une signification. Ce à quoi nous confronte

70. Cette expression de "salade de mots » fait encore partie de la terminologie sémiologique courante. C'est ainsi que, dans le DSM V, on peut lire que «le discours peut être si profondément désorganisé qu'il est presque incompréhensible et ressemble à la désorganisation linguistique de l'aphasie réceptive (incohérence ou "salade de mots")» (American Psychiatric Association(dir.), Diagnostic and statistical manual of mental disorders: DSM-5, p. 88, notre traduction).

71. Michel Cénac, «Logorrhée néologique chez une malade atteinte de délire hallucinatoire chronique à manifestations polymorphes », Bulletin de la société clinique de médecine mentale, 11, 1923, p. 70.

72. Ibid., p. 71.

73. Ibid., p. 70.

74. Ibid., p. 70. 
ainsi la «logorrhée néologique» d'Erneste, c'est donc à une suite de pseudo-mots qui, en tant qu'elle contredit les règles de la morphologie et de la syntaxe, ne peut pas même former une phrase. Dès lors, ce que nous nommons un « acte de langage vide » n'est pas seulement une phrase qui ne signifie rien dans les circonstances de son énonciation, mais une suite de sons telle que nous ne pouvons imaginer des circonstances où elle pourrait signifier quelque chose. De ces pseudo-phrases, il n'est pas même possible de dire qu'elles semblent contredire ce que nous tenons pour certain, car pour qu'une telle contradiction puisse être imaginée, il faudrait, au moins, que l'on puisse lui attribuer une signification possible.

Ce que nous enseigne alors ce détour par l'acte de langage vide c'est, par contraste, qu'on ne peut considérer un énoncé comme un «délire » qu'à condition qu'il remplisse les conditions minimales de la morphologie et de la syntaxe. Si dire, comme Mme de Rio-Branco, que « au fur et à mesure qu'ils m'enlevaient une enfant, ils m'en donnaient une autre qui lui ressemblait ${ }^{75}$ peut être considéré comme délirant, c'est dans la mesure où, à la différence des actes de langage vides, il est possible d'imaginer des circonstances où cette phrase pourrait avoir un sens. Ce qui ne signifie nullement que le délire posséderait un sens, une structure morphologique et syntaxique minimale ne suffisant pas, nous l'avons vu, à un écarter la possibilité d'un non-sens. Cela implique seulement que si le délire suppose d'être suffisamment structuré pour qu'on puisse lui attribuer par l'imagination une signification, c'est seulement en tant qu'il est le Doppelgänger d'une proposition qui, dans les circonstances de son énonciation, fait sens. On ne parle de délire, pour dire les choses autrement, qu'à partir du moment où ce dernier possède l'apparence d'une croyance, sans pouvoir être considéré, pour autant, comme une croyance.

\subsection{Croire que l'on croit}

Cette ressemblance superficielle entre croyance et délire ne saurait toutefois suffire à mettre en évidence le fossé qui sépare ce dernier d'un «acte de langage vide». De fait, on oublie parfois que cette proximité est déjà observée par Berrios lui-même, ce dernier notant que si le délire n'est pas une « croyance », il n'en est pas moins « un "discours vide" déguisé en croyance $\gg{ }^{76}$. Et pourtant, il y a encore quelque chose d'insatisfaisant dans cette manière de décrire le délire, qui passe à côté de ce qui le distingue d'un acte de langage vide, même "déguisé en croyance ». On notera, pour commencer, que l'intention de Berrios, lorsqu'il définit le délire comme un acte de langage vide, est de montrer que, dans la mesure où il ne dit rien sur le «monde » ou sur le «soi », alors « la seule information qu'il pourrait transporter doit être rattachée à des événements neurobiologiques ${ }^{77}$. De ce

75. Capgras, op. cit., p. 9.

76. Berrios, op. cit., p. 91.

77. Ibid., p. 8, nous soulignons. 
point de vue, les mots et les phrases prononcés par le sujet ne sont rien d'autre que « du bruit psychosocial dans lequel [le délire] s'enveloppe $»^{78}$.

Or comme nous allons tenter de le montrer, considérer que le délire est un «non-sens » n'implique nullement de réduire la parole du sujet délirant à un simple «bruit » qui pourrait, au mieux, être expliqué par des causes neurobiologiques. Certes, il est important de souligner, à la suite des lectures « austères » de Wittgenstein ${ }^{79}$, qu'aucune compréhension du non-sens en tant que tel ne saurait être admise, le non-sens ne pourrait être quelque chose que l'on pourrait «croire» ou «penser». Cependant, comme l'a défendu Cora Diamond, si suggérer une compréhension du non-sens est déjà un non-sens, il n'en reste pas moins possible de « comprendre une personne qui prononce un non-sens ${ }^{80}$. Afin de préciser ce qu'implique une telle forme de compréhension, la philosophe la distingue à la fois de la «compréhension intérieure », qui appréhende ce qui est dit à partir des lois de la logique, et de la «compréhension extérieure », qui appréhende ce qui est dit à partir des lois de la nature. Comprendre la personne qui prononce un non-sens ne saurait nous faire entrer à "l'intérieur» de l'énoncé, puisque ce dernier est contraire à notre grammaire. Cependant, appréhender le non-sens de "l'extérieur», en observant par exemple dans quelles circonstances et suivant quelles lois naturelles les mots sont prononcés, nous permet seulement d'expliquer pourquoi il a été prononcé, sans pour autant le comprendre. Ce qui est l'objet de notre compréhension, observe alors Diamond, c'est bien plutôt «l'inclination » du sujet à " prendre le non-sens pour du sens » c'est-à-dire à " penser qu'il est en train de penser quelque chose $»^{81}$ ou, pourrions-nous ajouter, à croire qu'il est en train de croire quelque chose.

Ce point est souligné par Wittgenstein dans le Big Typescript, où il note que la philosophie n'implique aucun renoncement «intellectuel» puisqu'en écartant les phrases qui constituent des non-sens, « nous ne nous réfrénons pas de dire quelque chose, mais abandonnons plutôt une certaine combinaison de mots comme vide de sens ${ }^{82}$. En revanche, et c'est là le point important, elle suppose bien un renoncement «affectif» (offeeling) car « il peut être aussi difficile de ne pas employer une expression, que de retenir des larmes, ou une explosion de colère ${ }^{83}$. De là découle une

78. Ibid., p. 8, nous soulignons.

79. L'interprétation « austère » du non-sens vise, à la suite des travaux de Cora Diamond et James Conant, de mettre l'accent sur la radicalité de la proposition wittgensteinienne : le nonsens n'est pas une phrase dont la signification serait fausse ou partielle, mais une phrase dépourvue de toute signification.

80. Cora Diamond, «Ethics, Imagination and the Method of Wittgenstein's Tractatus », in Alice Crary et Rupert J. Read (dir.), The New Wittgenstein, Routledge, 2000, p. 157, nous soulignons, notre traduction.

81. Ibid., p. 156.

82. Wittgenstein, The Big Typescript, TS 213, trad. C. Grant Luckhardt et Maximilian A.E. Aue, German-English scholars'ed., Malden, MA, Blackwell, 2005, p. 301, notre traduction française.

83. Ibid., p. 301. 
distinction, qui nous semble en mesure d'éclairer la question de la nature doxastique du délire, à savoir que si une personne qui prononce un non-sens ne peut pas «croire ce qu'elle dit», il n'en reste pas moins qu'elle peut être tentée de « croire qu'elle croit ce qu'elle dit».

Soutenir ainsi que le délire implique de «croire que l'on croit» peut sembler être une thèse paradoxale, allant à l'encontre de la démarche grammaticale qui vise habituellement à mettre en lumière « une réponse qui paraît triviale et qui, en effet, est triviale $»^{84}$. Mais si une telle compréhension du délire peut sembler étrangement tautologique, c'est seulement du fait de la formulation concise que nous en proposons. Car dire de quelqu'un qu'il « croit croire ce qu'il dit» est, à y regarder de plus près, une simple description de l'acte consistant à prononcer un non-sens. Pour mettre ce point en évidence il est nécessaire de préciser en quoi consiste cette « inclination» à prendre du non-sens pour du sens. Les raisons que quelqu'un peut avoir de croire qu'il dit quelque chose peuvent être, pour commencer, "subjectives », au sens où le sujet du délire peut avoir des motifs affectifs et personnels de dire ce qu'il dit. Les mots employés peuvent présenter des sonorités, ou être associés à des significations, telles que le sujet est enclin à les prononcer. Cependant, de telles inclinations sont tout aussi présentes dans le délire que dans l'acte de langage vide. On peut, en effet, expliquer la suite de phonèmes prononcée par Erneste par la préférence que l'on peut avoir envers une sonorité plutôt qu'une autre. Ce que l'on observe toutefois dans le délire, et dans le délire seulement, c'est ce que l'on pourrait nommer des raisons " objectives », au sens où il y a quelque chose dans la forme même du non-sens qui nous incline à le prononcer. Cette inclination, nous l'avons déjà observé, repose sur la ressemblance qui existe entre le non-sens et ses Doppelgänger doués de sens, avec lesquels nous le confondons parfois. Ce que le délire peut avoir, à cet égard, de particulièrement troublant, c'est précisément le fait qu'une phrase telle que « ma fille a été remplacée par un sosie » puisse être imaginée comme ayant un sens dans d'autres circonstances. Cela créé une illusion grammaticale, qui nous amène à croire que lorsque nous disons « je ne suis pas sûr que mes parents soient mes parents », le fait de prononcer ces paroles suffit à exprimer un doute. C'est cette relation de similitude interne entre différents Doppelgänger qui fait que nous pouvons parfois croire que nous disons quelque chose alors que nous ne disons rien et, par là même, croire que nous croyons quelque chose.

On notera, pour conclure, que réintroduire la notion de «croyance» n'implique nullement de revenir à une conception doxastique du délire, au contraire. Ce que nous avons tenté mettre en évidence, c'est bien plutôt que l'observation clinique suivant laquelle le patient semble croire ce qu'il dit ne nous contraint nullement à admettre que l'attribution d'un délire implique l'attribution d'une croyance. Et pour cause, il y a un fossé entre l'attitude qui

84. Vincent Descombes, Le Complément de sujet : enquête sur le fait d'agir de soi-même, 2018, p. 12. 
consiste à croire ce que nous disons, qui suppose d'employer une phrase dans un contexte conforme à une norme, et celle qui consiste à croire que nous croyons ce que nous disons, qui suppose seulement une similitude superficielle entre un non-sens et une phrase douée de sens. De ce point de vue, l'erreur qui se trouve à la racine des conceptions doxastiques est de s'être laissé piéger par l'illusion grammaticale propre au délire, où l'apparence d'une croyance n'a pas valeur de croyance.

\section{Conclusion}

$\mathrm{Au}$ cœur du débat opposant les conceptions «doxastiques » et «non-doxastiques» se trouve la question des relations entre philosophie et psychopathologie. En effet, si la question de savoir si le délire est une croyance semble faire sens, c'est dans la mesure où les philosophes endossent ce qu'ils semblent considérer comme un fait clinique, à savoir que le sujet délirant croit ce qu'il dit, même lorsque ce qu'il dit va à l'encontre de nos propres certitudes. Cependant, comme nous avons tenté de le montrer, s'il existe une proximité grammaticale entre délire et croyance, que l'on retrouve nécessairement dans les observations du clinicien, cela ne signifie nullement que nous devons rendre compte du délire comme étant une croyance. On doit, sur ce point, à Wittgenstein d'avoir mis en évidence qu'une phrase contredisant ce que nous tenons pour certain ne peut être qu'un non-sens ayant l'apparence d'un sens. C'est parce qu'une telle phrase possède des Doppelgänger, c'est-à-dire des phrases possédant la même grammaire de surface, qu'elles peuvent sembler faire sens là où elles échouent à le faire. Et c'est cette même proximité superficielle qui créé l'illusion que nous disons, pensons, croyons quelque chose, alors même que nous ne faisons que prononcer des non-sens. Or il n'en va pas différemment dans le cas du délire, où l'on peut admettre que le sujet délirant croit qu'il croit ce qu'il dit, et peut avoir des raisons de le croire, sans conclure pour autant que ce qu'il peut être l'objet légitime d'une croyance. Arriver à une telle conclusion, ce n'est pas pour autant rejeter tout dialogue entre philosophie et psychopathologie ; c'est au contraire souligner que le point de départ de ce dernier ne doit pas être ce que l'on pourrait considérer, de manière imprudente, comme une évidence clinique, mais bien plutôt les illusions grammaticales que philosophes, psychologues et psychiatres sont enclins à partager. 